(c) 2018 IEEE. Personal use of this material is permitted. Permission from IEEE must be obtained for all other uses, in any current or future media, including reprinting/republishing this material for advertising or promotional purposes, creating new collective works, for resale or redistribution to servers or lists, or reuse of any copyrighted component of this work in other works.

Digital Object Identifier (DOI): 10.1109/ECCE.2016.7855562

IEEE Energy Conversion Congress and Exposition (ECCE), Milwaukee, WI, 2016

Voltage stability analysis using a complete model of grid-connected voltage-source converters

Zhi-Xiang Zou

Giampaolo Buticchi

Marco Liserre

Andreas Martin Kettner

Mario Paolone

Suggested Citation

Z. Zou, G. Buticchi, M. Liserre, A. M. Kettner and M. Paolone, "Voltage stability analysis using a complete model of grid-connected voltage-source converters," in IEEE Energy Conversion Congress and Exposition (ECCE), Milwaukee, WI, 2016, pp. 1-8. 


\section{Voltage Stability Analysis Using A Complete Model of Grid-Connected Voltage-Source Converters}

\author{
Zhixiang Zou, Giampaolo Buticchi, Marco Liserre \\ Christian-Albrechts-Universiät zu Kiel \\ Chair of Power Electronics \\ Kiel, Germany \\ E-Mail: $\{z z$, gibu,ml $\} @$ tf.uni-kiel.de
}

\author{
Andreas Martin Kettner, Mario Paolone \\ École Polytechnique Fédérale de Lausanne \\ Distributed Electrical Systems Laboratory \\ Lausanne, Switzerland \\ E-Mail: \{andreas.kettner,mario.paolone\}@epfl.ch
}

\begin{abstract}
Due to the increasing popularity of renewable energies, a significant share of the power generation in future microgrids is expected to originate from converter-interfaced Distributed Energy Resources (DERs). Traditionally, idealized device models are used to conduct grid stability studies. For instance, a DER interfaced via a Voltage-Source Converter (VSC) would be modeled as an ideal current or power source (depending on the control schemes), ignoring non-ideal behavior like the response of the converter synchronization. However, such a simplification may lead to misjudging the stability, in particular for weak microgrids. To address this issue, ZIP models of grid-interfaced VSCs, which take into account both the control scheme and the synchronization, are developed in this paper. The influence of the synchronization response on the stability of a weak microgrid system is demonstrated using a benchmark system simulated in MATLAB/Simulink. It is shown that the idealized models normally used for static stability analysis do underestimate the voltage stability issue in the investigated microgrid system.

Index Terms-microgrids, voltage collapse, Distributed Energy Resources (DERs), polynomial load model, grid-interfaced inverter control, grid synchronization
\end{abstract}

\section{INTRODUCTION}

The increasing use of Voltage Source Converter (VSC) interfaced Distributed Energy Resources (DERs) introduces new challenges in assessing the stability of microgrids [1]. In particular, stability is a major concern when a weak system is operated under stressed conditions, for instance close to the point of voltage collapse. Under such circumstances, the system is more vulnerable because even a slight disturbance may lead to complete collapse.

Various approaches have been proposed to assess voltage collapse, for instance methods based on continuation power flow [2], pairs of load flow solutions [3], closest bifurcation points [4], indices computed from the singular values of the load flow Jacobian [5], and loadability limits [6]. Up to now, most investigations concerning static voltage stability have been focused on high voltage transmission grids. However, this subject has received little attention for distribution grids in general and low-voltage microgrids in particular.

In this context, the modeling of DERs is one of the key issues for the voltage stability analysis in low-voltage electric networks. In most of the literature [7], DERs interfaced via VSCs are modeled either as ideal current sources or as PQ nodes, considering only the current/power control loop. However, the converters' synchronization is seldom accounted. Such models are valid when the voltage at the Point of Common Coupling (PCC) is maintained close to its nominal value. Under this condition, these ideal models can be used to assess different stability issues of VSCs, for example steady-state small-signal stability [8], and harmonic stability [9]. However, further investigations show that the synchronization algorithm may lead the system to instability in certain cases [10]. The input-admittance of the VSC can be significantly changed especially when reactive power control is performed. Furthermore, an impedance model of VSC with synchronization has been developed, illustrating the effects of synchronization on the VSC in mathematics model and analysis [11]. This methodology improves the model accuracy and can be used to assess system-level compatibility and power quality. In addition, the influence of synchronization on the small-signal stability of VSC has been studied in [12], showing that the characteristics of PLL play an important role on the system stability.

In this paper, ZIP models are developed for both the power- and current-controlled VSC-interfaced DERs with Phase-Locked Loop (PLL)-based synchronization. It is shown that the ZIP model (especially the constant current and power parts) can be affected by the characteristics of PLL, for example its bandwidth. The developed models can be applied to the voltage stability analysis to achieve more accurate assessment. The rest of the paper is organized as follows: the voltage instability and loadability limit are presented in Section II. The ZIP models of current/power controlled VSC with PLLs are developed in Section III. Section IV studies the behavior of a benchmark microgrid when different PLLs are used in the system, showing that the PLL affects the margin of voltage stability in a stressed microgrid. Section V draws the conclusions.

\section{Voltage Collapse}

For an electrical grid modeled with a phasor-based approach, [13] has defined the voltage collapse as follows: "voltage instability stems from the attempt of the load dynamics to restore power consumption beyond the capability of the combined transmission and generation system". The critical point where instability occurs can be determined from $P V$ 
curves [14], which can be derived using Continuation Power Flow (CPF) methods [2]. Unfortunately, CPF methods are computationally demanding because they are based on an iterative procedure which requires solving a Load Flow (LF) problem in each step. However, if one is only interested in determining the margin from the current point of operation to the point of voltage collapse, but not in behavior of the system in the intermediate region, more light-weight methods based on nonlinear programming can be employed [6].

\section{A. Network Equations}

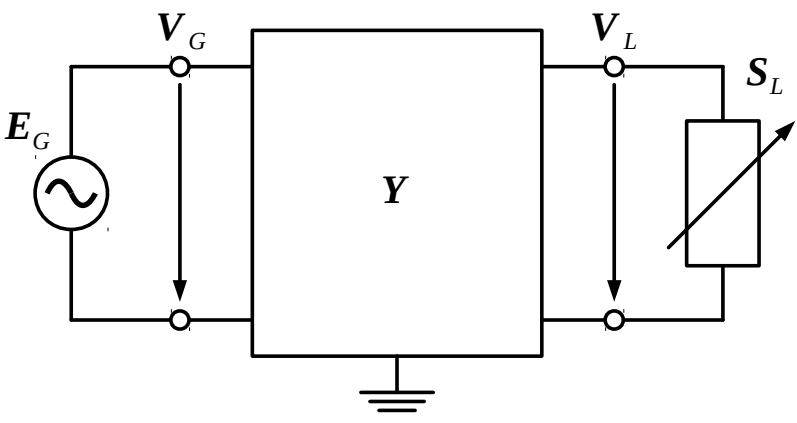

Fig. 1. Network consisting of generator buses $\mathcal{G}$ (modeled as ideal voltage sources $\mathbf{E}_{G}$ ) and load buses $\mathcal{L}$ (modeled as polynomial loads $\mathbf{S}_{L}$ ) interconnected by a network with admittance matrix $\mathbf{Y}$. The vectors of nodal voltage phasors are denoted by $\mathbf{V}_{G}$ and $\mathbf{V}_{L}$, respectively.

Consider the network shown in Figure 1, whose buses $\mathcal{N}$ can be grouped into generator buses $\mathcal{G}$ modeled as voltage sources $\mathbf{E}_{G}$, and load buses $\mathcal{L}$ modeled as voltage-dependent injections $\mathbf{S}_{L}$. The network is described by the nodal admittance matrix $\mathbf{Y}$, which links the nodal voltages $\mathbf{V}_{G}, \mathbf{V}_{L}$ with the nodal currents $\mathbf{I}_{G}, \mathbf{I}_{L}$.

$$
\left[\begin{array}{c}
\mathbf{I}_{G} \\
\mathbf{I}_{L}
\end{array}\right]=\left[\begin{array}{ll}
\mathbf{Y}_{G G} & \mathbf{Y}_{G L} \\
\mathbf{Y}_{L G} & \mathbf{Y}_{L L}
\end{array}\right]\left[\begin{array}{c}
\mathbf{V}_{G} \\
\mathbf{V}_{L}
\end{array}\right]
$$

Without loss of generality, it may be assumed that the network is Kron-reduced, that is any tie buses $\mathcal{T}$ without injection have been eliminated from $\mathbf{Y}$ [15]. Under these assumptions, the nodal injections $S_{L, k}(k \in \mathcal{L})$ are given by

$$
\begin{aligned}
S_{L, k} & =V_{L, k} I_{L, k}^{*} \\
& =V_{L, k}\left(\operatorname{row}_{k}\left(\mathbf{Y}_{L G}^{*}\right) \mathbf{E}_{G}^{*}+\operatorname{row}_{k}\left(\mathbf{Y}_{L L}^{*}\right) \mathbf{V}_{L}^{*}\right)
\end{aligned}
$$

where $I_{L, k}^{*}$ is substituted from (1), and obviously $\mathbf{V}_{G}=\mathbf{E}_{G}$. Note that $(.)^{*}$ denotes the complex conjugate.

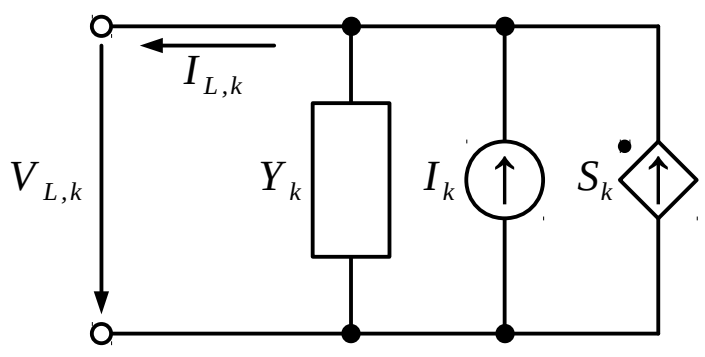

Fig. 2. Polynomial load model (also known as ZIP).
In this work, the voltage-dependent loads $S_{L, k}$ are described by polynomial models [13]. A polynomial load is composed of a constant admittance $Y_{k}$, a constant current source $I_{k}$ (magnitude $\left|I_{k}\right|$, power factor $\cos \left(\vartheta_{k}\right)$ ), and constant power source $S_{k}$ as shown in Fig. 2. So, $S_{L, k}$ is given by

$$
S_{L, k}=-Y_{k}^{*}\left|V_{L, k}\right|^{2}+\left|V_{L, k}\right|\left|I_{k}\right| \angle \vartheta_{k}+S_{k}
$$

where $\left|V_{L, k}\right|$ is the magnitude of the phasor $V_{L, k}=$ $\left|V_{L, k}\right| \angle \delta_{L, k}$. In general, a polynomial load model is only be an approximation of a general function $S_{L, k}\left(V_{k}\right)$, namely a second order Talyor series with coefficients $S_{k}, I_{k}$, and $-Y_{k}^{*}$. In high-voltage transmission systems, ZIP models are an approximation since they model aggregated resources. For the particular case of microgrids, these coefficients do have a physical counterpart, namely converters with constant power or constant current control, and passive loads. Hence, the polynomial model is an exact representation of load and generator (controlled in power or current mode) and not just a mere approximation.

Obviously, the injection from the point of view of the network (3) and of the load (4) have to be equal. Hence, for fixed values of $\mathbf{V}_{G}$ the load flow equations can be written in compact form as

$$
\varphi(\nu)=0
$$

where $\boldsymbol{\nu}$ denotes the state vector of the network, and is composed of the voltage magnitudes $\left|V_{L, k}\right|$ and phase angles $\delta_{L, k}$ of the load buses. The functions $\varphi_{k}(\boldsymbol{\nu})$ capture that (3) and (4) are equal $\forall k \in \mathcal{L}$

$$
\varphi_{k}(\boldsymbol{\nu})=\left\{\begin{array}{c}
\operatorname{row}_{k}\left(\mathbf{Y}_{L G}^{*}\right) \mathbf{E}_{G}^{*}+\operatorname{row}_{k}\left(\mathbf{Y}_{L L}^{*}\right) \mathbf{V}_{L}^{*} \\
-\left(-Y_{k}^{*}\left|V_{L, k}\right|^{2}+\left|V_{L, k}\right|\left|I_{k}\right| \angle \vartheta_{k}+S_{k}\right)
\end{array}\right\}
$$

\section{B. Grid Loadability}

The point of voltage collapse can be determined by increasing the loads $S_{L, k}$ until the load flow (5) becomes unsolvable [6]. In principle, each component of the polynomial load (4) could be scaled independently in different areas of the network or at each individual bus. For the sake of simplicity, it is assumed that all loads are increased equally by a scaling factor $\sigma \in \mathbb{R}^{+}$

$$
S_{L, k}=\sigma\left(-Y_{k}\left|V_{L, k}\right|^{2}+\left|V_{L, k}\right|\left|I_{k}\right| \angle \vartheta_{k}+S_{k}\right)
$$

Including the $\sigma$ into the load flow equations (5) yields

$$
\varphi(\sigma, \nu)=0
$$

To determine the loadability limit, one would like to find the largest value $\bar{\sigma} \in \mathbb{R}^{+}$for which (8) remains solvable, which can be formulated as a Nonlinear Program (NLP)

$$
\begin{aligned}
& \min _{\sigma, \boldsymbol{\nu}} \zeta(\sigma) \\
& \text { s.t. } \boldsymbol{\varphi}(\sigma, \boldsymbol{\nu})=\mathbf{0}
\end{aligned}
$$

where $\zeta(\sigma)=-\sigma$. The above problem complies with the standard form of an equality-constrained NLP required by standard solvers (for instance the fmincon solver of MATLAB). 


\section{MODELING OF PLL-SYNCHRONIZED GRID-CONNECTED INVERTER}

The inverter interfacing a DER with the grid (shown in Fig. 3) can be operated either as a current source or as a power source which tracks a given reference setpoint (see [7], [16][19]) Both the current and the control scheme are essentially based on regulating the output current $\left[i_{a}, i_{b}, i_{c}\right]^{T}$, given the PCC voltage $\left[v_{a}, v_{b}, v_{c}\right]^{T}$. The average model of the system is

$$
L_{f} \frac{\mathrm{d}}{\mathrm{d} t}\left[\begin{array}{c}
i_{a} \\
i_{b} \\
i_{c}
\end{array}\right]=G_{P W M}\left[\begin{array}{l}
v_{r a} \\
v_{r b} \\
v_{r c}
\end{array}\right]-\left[\begin{array}{c}
v_{a} \\
v_{b} \\
v_{c}
\end{array}\right] .
$$

where $L_{f}$ is the output filter of the grid inverter, $\left[v_{r a}, v_{r b}, v_{r c}\right]^{T}$ are the modulating references of the grid inverter, and $G_{P W M}$ represents the delay and gain of the Pulse-Width Modulation (PWM).

\section{A. Linearization of PLL Synchronization}

In this paper, a three-phase PLL is employed to determine the phase angle of PCC voltage, which is is assumed symmetrical. Its detailed block diagram is shown in Fig. 4. A detailed small-signal model is derived in the following in order to study the characteristics of the PLL.

Consider a small perturbation on phase $a$ of the PCC voltage

$$
v_{a}(t)=V \cos \left(\omega_{0} t\right)+\Delta V\left(\omega_{p} t+\phi_{p}\right)
$$

where $V$ and $\omega_{0}$ are the amplitude and the frequency of the PCC voltage, and $\Delta V$ and $\omega_{p} t+\phi_{p}$ are the amplitude and the phase of the small-signal perturbation. Assuming that the PLL is implemented under stable conditions, namely the detected frequency is intentionally frozen at $\omega$ which is slightly different from $\omega_{0}$, i.e. $\omega \approx \omega_{0}$. The phase displacement between the PCC voltage phase angle $\theta_{0}$ and the output of $\operatorname{PLL}(\theta)$ is $\Delta \theta$.

The PCC voltage can be transformed from the $a b c$ frame to the $d q 0$ frame using Park's transformation. Since the PCC voltage is assumed symmetric, the zero component is simply null. In order to linearize the transformation, the transfer matrix is broken into two parts

$$
\begin{aligned}
\mathbf{T}(\theta) & =\left(\begin{array}{ccc}
\cos \theta & \cos (\theta-2 \pi / 3) & \cos (\theta+2 \pi / 3) \\
-\sin \theta & -\sin (\theta-2 \pi / 3) & -\sin (\theta+2 \pi / 3) \\
0.5 & 0.5 & 0.5
\end{array}\right) \\
& =\left(\begin{array}{ccc}
\cos \Delta \theta & \sin \Delta \theta & 0 \\
-\sin \Delta \theta & \cos \Delta \theta & 0 \\
0 & 0 & 1
\end{array}\right) \mathbf{T}\left(\theta_{0}\right)
\end{aligned}
$$

where $\theta=\theta_{0}+\Delta \theta$ denotes the output angle of the PLL, and $\theta_{0}=\omega_{0} t$ is the phase of actual PCC voltage. Using Park's transformation, the $q$-axis voltage $v_{q}^{\prime}(t)$ can be calculated as

$$
\begin{aligned}
v_{q}^{\prime}(t) & =-\sin \Delta \theta(t) \cdot v_{d}(t)+\cos \Delta \theta(t) \cdot v_{q}(t) \\
& \approx-\Delta \theta(t) \cdot v_{d}(t)+v_{q}(t)
\end{aligned}
$$

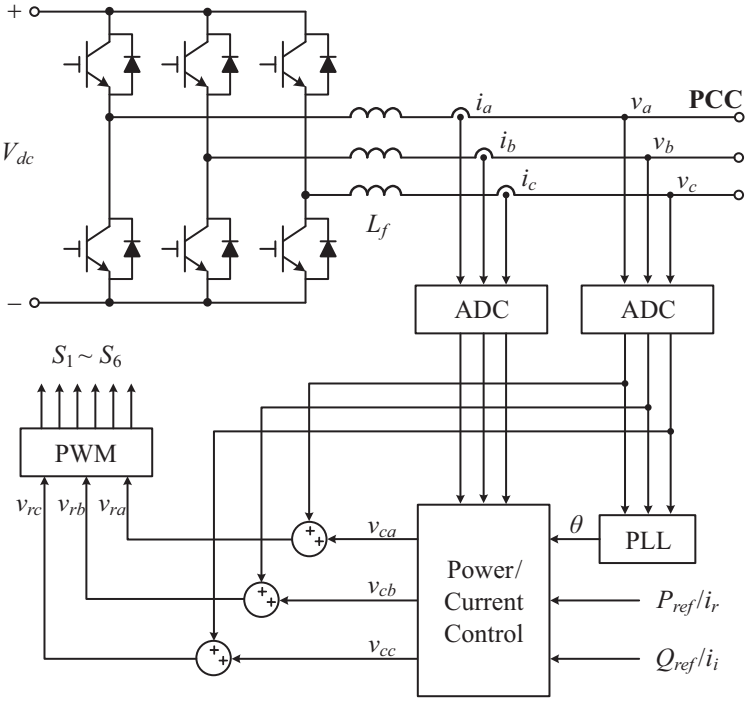

Fig. 3. Control system of a three-phase grid-interfaced inverter for DER.

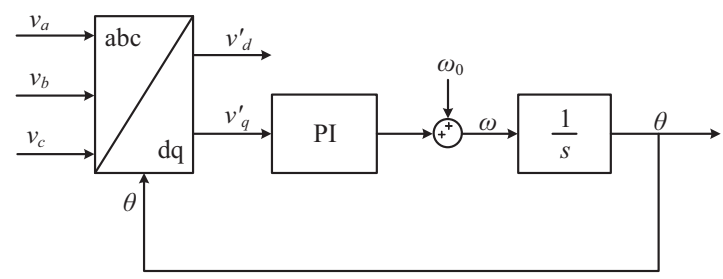

Fig. 4. Diagram of three-phase PLL.

where $v_{d}(t)$ and $v_{q}(t)$ are the $d$ - and $q$-axis voltage obtained by applying $\mathbf{T}\left(\theta_{0}\right)$, namely

$$
\left(\begin{array}{l}
v_{d}(t) \\
v_{q}(t)
\end{array}\right)=\left(\begin{array}{l}
V \\
0
\end{array}\right)+\left(\begin{array}{c}
e^{j \omega_{0} t} \Delta \underline{V} \\
-j e^{j \omega_{0} t} \Delta \underline{V}
\end{array}\right)
$$

Combining (13) and (14) yields the small-signal variation $\Delta v_{q}^{\prime}(t)$ of $v_{q}^{\prime}(t)$ as

$$
\Delta v_{q}{ }^{\prime}(t)=-\Delta \theta(t) \cdot V-j e^{j \omega_{0} t} \cdot \Delta \underline{V}
$$

where $\Delta \underline{V}$ is the small-signal variation of the three-phase PCC voltage in frequency domain.

From Fig. 4, it can be seen that $\Delta \theta(t)=G_{i n} \cdot \Delta v_{q}{ }^{\prime}(t)$, where $G_{i n}$ is the response from $v_{q}{ }^{\prime}(t)$ to $\theta(t)$ in time-domain. By substituting (15) into this relation and applying the Laplace transformation, one obtains the transfer function of the PLL in $s$-domain

$$
G_{P L L}{ }^{\prime}(s)=\mathscr{L}\left\{\frac{\Delta \theta(t)}{\Delta v(t)}\right\}=\frac{\Theta(s)}{V(s)}=\frac{-j G_{i n}\left(s-j \omega_{0}\right)}{1+V G_{i n}\left(s-j \omega_{0}\right)}
$$

The current references in phase-domain are calculated based on the detected phase of the three-phase PLL. take the current reference of phase-A as example, $i_{\text {ref_a }}{ }^{\prime}(t)=I_{\text {ref_a }} a$. $\cos \left(\omega_{0} t+\Delta \theta(t)\right)$, and it can be rewritten as

$$
i_{\text {ref_a }}{ }^{\prime}(t)=I_{\text {ref_a }} \cos \left(\omega_{0} t+\Delta \theta(t)\right)=i_{\text {ref }}(t)+\Delta i_{\text {ref }}(t)
$$


where $I_{r e f_{-} a}$ is the amplitude of reference current for phase $a$, and $i_{r e f}(t)=I_{r e f_{-} a} \cos \left(\omega_{0} t\right)$ is its large-signal AC component. The small-signal perturbation of $i_{\text {ref }}{ }^{\prime}(t)$ is

$$
\Delta i_{r e f_{-} a}{ }^{\prime}(t) \approx-I_{r e f_{-} a} \Delta \theta(t) \sin \left(\omega_{0} t\right)
$$

As a result, the transfer function from the phase angle of PLL to the current reference is

$$
G_{r e f}(s)=\mathscr{L}\left\{\frac{\Delta i_{r e f}(t)}{\Delta \theta(t)}\right\}=\frac{I_{r e f}(s)}{\Theta(s)}
$$

which can be rewritten as

$$
I_{r e f}(s)=\frac{I_{r e f \_} a}{2 j}\left(\Theta\left(s-j \omega_{0}\right)-\Theta\left(s+j \omega_{0}\right)\right)
$$

The relation between the PCC voltage and the current reference can be established by combining (16) and (20)

$$
\begin{aligned}
G_{P L L}(s) & =\frac{I_{r e f_{-} a}}{2} \cdot\left(\frac{G_{P L L}{ }^{\prime}(s)}{1+V G_{P L L}{ }^{\prime}(s)}\right. \\
& \left.-\frac{G_{P L L}{ }^{\prime}\left(s-j 2 \omega_{0}\right)}{1+V G_{P L L}{ }^{\prime}\left(s-j 2 \omega_{0}\right)}\right)
\end{aligned}
$$

which, by neglecting the second-order terms of the perturbation, reduces to

$$
G_{P L L}(s)=\frac{I_{r e f \_a}}{2} \cdot \frac{G_{P L L}{ }^{\prime}(s)}{1+V G_{P L L}{ }^{\prime}(s)}
$$

Due to the symmetry of the system, the transfer functions of the PLLs in phases $b$ and $c$ are the same as (22). The frequency responses of $G_{P L L}(s)$ with different bandwidths are presented in Fig. 4. One can see that the PLL with lower bandwidth introduces a magnitude deviation and a phase lag into the control system, which compromises the control accuracy. For voltage stability, the magnitude deviation and phase lag at $50 \mathrm{~Hz}$ could alter the constant-current and power sources and the power factor of (4). As a result, the ZIP models taking into account the PLL would be different from the idealized polynomial model. The stability margin would be significantly changed according to the characteristics of PLLs in the distribution network.

\section{B. ZIP Model of Grid-Interfaced Inverters}

The current control scheme is illustrated in Fig. 6(a), in which the voltage orientation is determined using the phase angle $\theta$ obtained from synchronization. Ideally, the fundamental component of the current vector is supposed to be locked to the voltage vector, so that the inverter can accurately inject, or absorb, the requested current/power. The output currents of the grid inverter are measured and sampled through an Analog-toDigital Converters (ADC) and passed into the controller. $I_{r e f_{r} r}$ and $I_{r e f_{-} i}$ are the real part and imaginary part of the reference current. Using Park's transformation, the reference currents can be computed in phase domain. Since the phase angle $\theta$ is measured by the PLL, the obtained reference currents highly depend on its characteristics. Note that the error between the phase measured by the PLL and the actual phase affects the reference currents both in amplitude and phase.

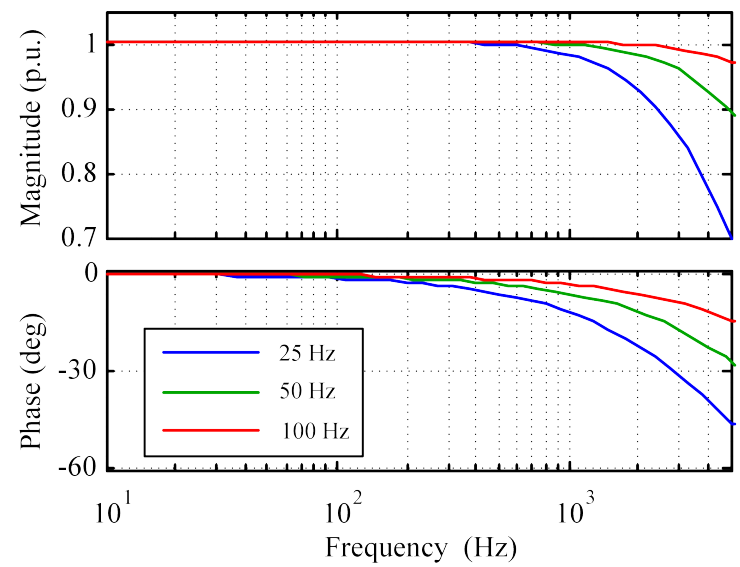

(a)
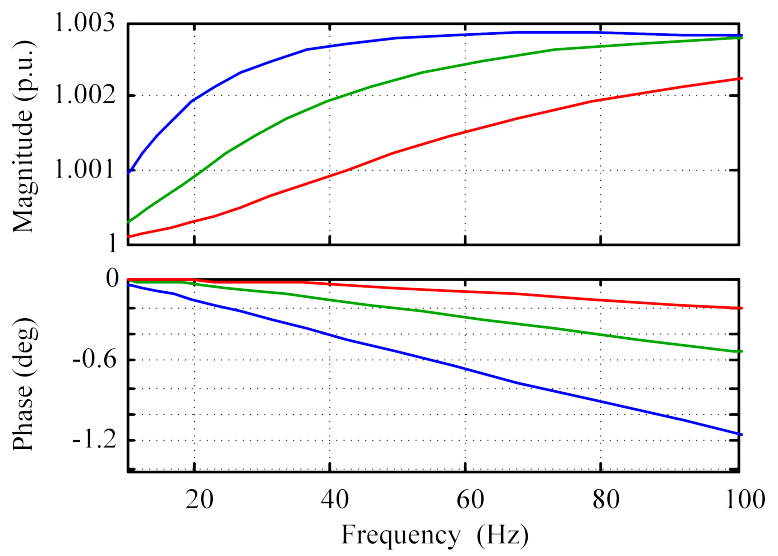

(b)

Fig. 5. Frequency responses of $G_{P L L}(s)$ with different bandwidths: (a) overview and (b) zoomed view at the neighboringhood of $50 \mathrm{~Hz}$.

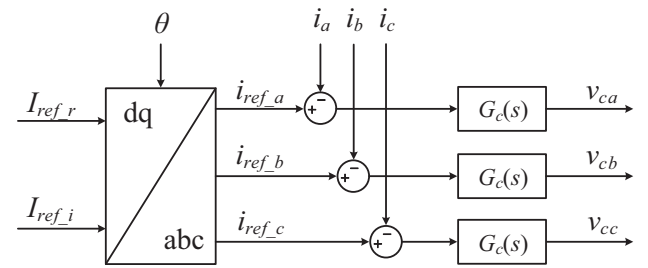

(a)
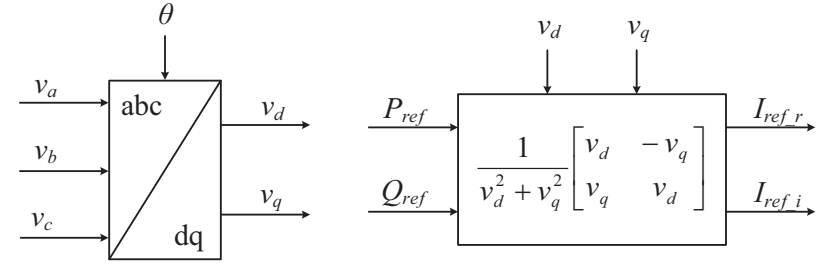

(b)

Fig. 6. Power/Current control schemes for grid inverter: (a) current control and (b) power control. 
Based on Fig. 3 and Fig. 6(a), the modulating references of the grid inverter can be determined as

$$
\begin{gathered}
{\left[\begin{array}{c}
v_{r a} \\
v_{r b} \\
v_{r c}
\end{array}\right]=-G_{c}(s) G_{A D C}(s)\left[\begin{array}{c}
i_{a} \\
i_{b} \\
i_{c}
\end{array}\right]+G_{c}(s)\left[\begin{array}{l}
i_{r e f_{-} a} \\
i_{r e f_{-} b} \\
i_{r e f_{-} c}
\end{array}\right]} \\
+G_{A D C}(s)\left[\begin{array}{l}
v_{a} \\
v_{b} \\
v_{c}
\end{array}\right] .
\end{gathered}
$$

where $\left[i_{\text {ref_a }}, i_{\text {ref_b }}, i_{\text {ref_c }}\right]^{T}$ are the the reference currents in phase-domain, and $G_{c}(s)$ and $G_{A D C}(s)$ is the transfer function of the current controller and the ADC. The ZIP model representing constant-current resources is obtained by substituting (23) into (10). Its components are listed below

$$
\begin{aligned}
Z_{k} & =\frac{L_{f} s+G_{c}(s) G_{A D C}(s) G_{P W M}(s)}{1-G_{A D C}(s) G_{P W M}(s)} \\
I_{k} & =\frac{G_{P W M}(s) G_{c}(s)}{L_{f} s+G_{c}(s) G_{A D C}(s) G_{P W M}(s)} \underline{I}_{r e f_{-} a b c} \\
S_{k} & =0
\end{aligned}
$$

where $\underline{I}_{r e f \_a b c}=\frac{1}{3}\left(i_{r e f_{-} a}+e^{j 120^{\circ}} i_{r e f_{-} b}+e^{j 240^{\circ}} i_{\text {ref_c }}\right)$ is dependent on the Park's transformation and the reference currents in $d q$-frame, which can be depicted by

$$
\underline{I}_{r e f_{-} a b c}=G_{P L L}\left(s-j \omega_{0}\right) \underline{I}_{r e f_{-} d q} \underline{V}_{a b c} .
$$

where $G_{P L L}(s)$ is the transfer function of the PLL from the PCC voltage to the phase of currents in phase-domain.

The control scheme for a power-controlled resource is illustrated in Fig. 6(b). It consists of two nested loops, where the outer is an open-loop $P Q$ control, and inner loop is the the current control from Fig. 6(a). The reference currents for the inner loop are calculated by the power outer loop in $d q$ frame using the following matrix

$$
\left[\begin{array}{c}
I_{r e f_{-} r} \\
I_{r e f_{-} i}
\end{array}\right]=\frac{1}{v_{d}^{2}+v_{q}^{2}}\left[\begin{array}{cc}
v_{d} & -v_{q} \\
v_{q} & v_{d}
\end{array}\right]\left[\begin{array}{l}
P_{r e f} \\
Q_{r e f}
\end{array}\right] .
$$

where $\underline{S}_{r e f}=P_{r e f}+j Q_{r e f}$ is the power reference of the grid inverter, $v_{d}$ and $v_{q}$ are the $d$ - and $q$-axis voltage in $d q$ frame. Here, $v_{d}$ and $v_{q}$ rely on Park's transformation and the characteristics of the PLL. As a result, the reference currents in power control scheme can be expressed by

$$
\underline{I}_{r e f_{-} d q}=\frac{1}{\|V\|^{2}} e^{-j \omega_{0} t}(1-j) \underline{V}_{a b c} \underline{S}_{r e f} .
$$

where $V$ is the amplitude of the PCC voltage. Considering the inner current loop, the ZIP model of power controlled inverter is obtained where the constant current part is $I_{k}=0$, and the constant impedance part is

$$
Z_{k}=\frac{L_{f} s+G_{c}(s) G_{A D C}(s) G_{P W M}(s)}{1-G_{A D C}(s) G_{P W M}(s)} .
$$

Substituting (29) into (25) and decoupling the PCC voltage, the constant power part can be calculated by

$$
\begin{aligned}
S_{k}= & \frac{G_{P W M}(s) G_{c}(s)}{L_{f} s+G_{C}(s) G_{A D C}(s) G_{P W M}(s)} \\
& \cdot G_{P L L}(s)(1-j) \underline{V}_{a b c} \underline{S}_{r e f} .
\end{aligned}
$$

Obviously, the constant impedance component of both current and power control schemes are independent of the synchronization. However, the constant current part and constant power part do depend on it. In fact, both the magnitude $\left(\left|I_{k}\right|\right.$ and $\left.\left|S_{k}\right|\right)$ and the power factor $\left(\cos \left(\vartheta_{k}\right)\right)$ deviate from the expressions of the idealized model. Hence, in a system with a lot of PLL-synchronized resources, using the idealized instead of the ZIP models developed above leads to a misassessment of the stability. This is particularly dangerous if the network is operated under stressed conditions (i.e. with low stability margin), since even slight disturbances may result in unstable behavior.

From Fig. 5(a) and Fig. 5(b), it is straightforward to observe that for the case of PLLs with high bandwidth, the assumptions made in the idealized model (i.e. magnitude $=1$ p.u., phase $=0 \mathrm{deg}$ ) are closely satisfied. Hence, the behavior of the actual system can be predicted also with PLL. However, the mismatches become significant when PLLs with low bandwidth are introduced into the system, which means that the stability cannot be assessed without taking the PLL into account.

\section{CASE STUdy}

\section{A. System Configuration}

Fig. 7 shows the microgrid system used for this case study, whose topology and the cable types are adopted from a benchmark network introduced in [20]. The lengths of the cables are prolonged to the values given in TABLE I to obtain a weak grid. Note that the maximum distance from the slack (from R01 to R18) is around $1 \mathrm{~km}$, which is realistic for a low voltage network. The electrical parameters of the different cable types are presented in TABLE II. Note that the lines' $R^{\prime} / X^{\prime}$ ratio is high, which is a characteristic feature of distribution grids.

First, a critical loading $\left\{Y_{C, k}, I_{C, k}, S_{C, k}\right\}(k \in \mathcal{R})$ for which voltage collapse occurs need to be determined. This can be accomplished using the method discussed in Section II-B. Namely, for a light load condition $\left\{Z_{0, k}, I_{0, k}, S_{0, k}\right\}(k \in \mathcal{R})$, (9) is solved to determine the critical scaling factor $\bar{\sigma}$ which marks the onset of voltage collapse The critical loading is

$$
\left(\begin{array}{c}
Y_{C, k} \\
I_{C, k} \\
S_{C, k}
\end{array}\right)=\bar{\sigma}\left(\begin{array}{c}
Y_{0, k} \\
I_{0, k} \\
S_{0, k}
\end{array}\right) \quad \forall k \in \mathcal{R}
$$

Confer to TABLE III for the numeric values determined for this case study. The values are given in per unit of the base $P_{b}=1 \mathrm{~kW}, V_{b}=230 \mathrm{~V}$. A close-to-critical loading can be obtained by downscaling the critical values by $\kappa<1$

$$
\left(\begin{array}{c}
Y_{k} \\
I_{k} \\
S_{k}
\end{array}\right)=\kappa\left(\begin{array}{c}
Y_{C, k} \\
I_{C, k} \\
S_{C, k}
\end{array}\right) \quad \forall k \in \mathcal{R}
$$

In this paper, $\kappa=0.98$ is chosen such that the system is heavily stressed, but sufficiently far from actually becoming unstable. Note that $I_{k}$ and $S_{k}$ are the reference values which the converters are tracking, and by which they converters 


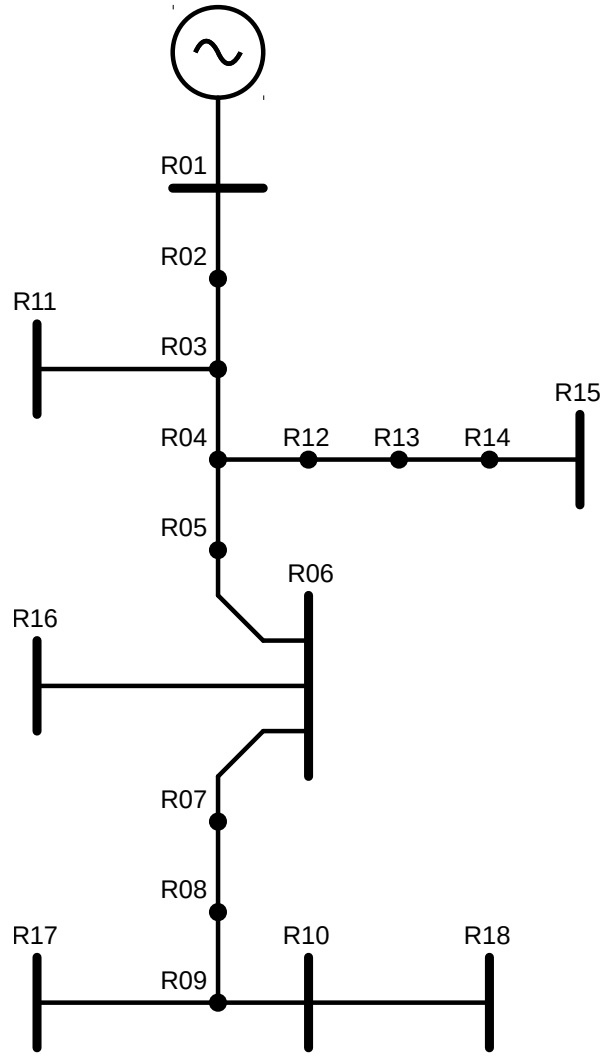

Fig. 7. Benchmark microgrid, adapted from [20]. Buses with generation or load are represented by bars $(\mathcal{G}=\{\mathrm{R} 01\}, \mathcal{L}=$ $\{\mathrm{R} 10-\mathrm{R} 11, \mathrm{R} 15-\mathrm{R} 18\})$, whereas tie buses are represented by circles $(\mathcal{T}=$ $\{\mathrm{R} 01-\mathrm{R} 04, \mathrm{R} 06-\mathrm{R} 09, \mathrm{R} 12-\mathrm{R} 14\})$.

would be represented using the idealized model. They are different from the values provided by the accurate model, which takes into account non-ideal effects.

Three VSC interfaced DERs are connected to the Buses of R15, R16, and R18, and their nominal power ratings are 101.98 p.u., 76.49 p.u., and 50.99 p.u., with Power Factor $(\mathrm{PF})=0.98$, respectively. Two VSC interfaced batteries are connected to the Buses of R6 and R10, both power ratings are 101.92 p.u., with $\mathrm{PF}=0.8$. Different types of loads are connected to the benchmark microgrid which are given in TABLE III.

The constant power and constant current sources/loads are controlled by using the power and current control schemes given in III-B. For both control schemes, a classic Proportional Resonant (PR) controller is selected and implemented in the current inner loop of each phase. The proportional and resonant gains of the PR controller are $K_{p}=5$ and $K_{r}=2000$, respectively. Three-phase PLLs have been applied for grid synchronization. The design of three-phase PLL follows the procedures mentioned in [21], according to which the PI parameters of PLL can be set as follows

$$
K_{p P L L}=2 \xi \omega_{n}=\frac{9.2}{t_{s}}, \quad T_{i P L L}=\frac{2 \xi}{\omega_{n}}=\frac{t_{s} \xi^{2}}{2.3} .
$$

TABLE I

LINE CONFIGURATION

\begin{tabular}{ccccc}
\hline Name & From & To & Cable & $L$ (m) \\
\hline \hline L-01 & R-01 & R-02 & UG-01 & 105 \\
L-02 & R-02 & R-03 & UG-01 & 105 \\
L-03 & R-03 & R-04 & UG-01 & 105 \\
L-04 & R-04 & R-05 & UG-01 & 105 \\
L-05 & R-05 & R-06 & UG-01 & 105 \\
L-06 & R-06 & R-07 & UG-01 & 105 \\
L-07 & R-07 & R-08 & UG-01 & 105 \\
L-08 & R-08 & R-09 & UG-01 & 105 \\
L-09 & R-09 & R-10 & UG-01 & 105 \\
L-10 & R-03 & R-11 & UG-04 & 90 \\
L-11 & R-04 & R-12 & UG-02 & 105 \\
L-12 & R-12 & R-13 & UG-02 & 105 \\
L-13 & R-13 & R-14 & UG-02 & 105 \\
L-14 & R-14 & R-15 & UG-02 & 90 \\
L-15 & R-06 & R-16 & UG-06 & 90 \\
L-16 & R-09 & R-17 & UG-04 & 90 \\
L-17 & R-10 & R-18 & UG-05 & 90 \\
\hline
\end{tabular}

TABLE II

CABLE TYPES

\begin{tabular}{cccc}
\hline Type & $R^{\prime}(\Omega / \mathrm{km})$ & $X^{\prime}(\Omega / \mathrm{km})$ & $R^{\prime} / X^{\prime}$ \\
\hline \hline UG-01 & 0.163 & 0.136 & 1.199 \\
UG-02 & 0.266 & 0.151 & 1.762 \\
UG-04 & 1.541 & 0.206 & 7.481 \\
UG-05 & 1.111 & 0.195 & 5.697 \\
UG-06 & 0.569 & 0.174 & 3.270 \\
\hline
\end{tabular}

TABLE III

Critical loAd (PER Phase, Balanced).

\begin{tabular}{ccccccc}
\hline Bus & $\begin{array}{c}\Re\left\{Y_{C, k}\right\} \\
(\mathrm{pu})\end{array}$ & $\begin{array}{c}\Im\left\{Y_{C, k}\right\} \\
(\mathrm{pu})\end{array}$ & $\begin{array}{c}\left|I_{C, k}\right| \\
(\mathrm{pu})\end{array}$ & $\begin{array}{c}\vartheta_{C, k} \\
(\mathrm{deg})\end{array}$ & $\begin{array}{c}P_{C, k} \\
(\mathrm{pu})\end{array}$ & $\begin{array}{c}Q_{C, k} \\
(\mathrm{pu})\end{array}$ \\
\hline \hline $\mathrm{R} 06$ & - & - & - & - & 81.54 & 61.15 \\
$\mathrm{R} 10$ & - & - & 102.09 & 31.95 & 81.54 & 61.15 \\
$\mathrm{R} 11$ & - & - & 50.960 & 36.87 & -86.63 & -54.02 \\
$\mathrm{R} 15$ & 0 & -25.48 & - & - & 101.92 & 0 \\
$\mathrm{R} 16$ & 50.96 & -50.96 & - & - & -86.63 & -54.02 \\
$\mathrm{R} 17$ & 0 & 20.38 & - & - & -86.63 & -54.02 \\
$\mathrm{R} 18$ & - & - & - & - & 0 & 31.60 \\
\hline
\end{tabular}


TABLE IV

COMPARISONS OF ZIP MODELS WITH DIFFERENT PLLS.

\begin{tabular}{ccccccc}
\hline & \multicolumn{5}{c}{$I_{k}$} & \multicolumn{2}{c}{$S_{k}$} \\
\cline { 2 - 7 } & ideal (no PLL) & fast PLL $(100 \mathrm{~ms})$ & slow PLL $(325 \mathrm{~ms})$ & ideal (no PLL) & fast PLL (100 ms) & slow PLL (325 ms) \\
\hline R06 & 0 & 0 & 0 & $79.9058+\mathrm{j} 59.9293$ & $79.8617+\mathrm{j} 59.8934$ & $79.2148+\mathrm{j} 59.6264$ \\
R10 & $84.8999+\mathrm{j} 52.9375$ & $84.8166+\mathrm{j} 53.0709$ & $84.8179+\mathrm{j} 53.0685$ & $79.9058+\mathrm{j} 59.9293$ & $79.8525+\mathrm{j} 59.8784$ & $79.0428+\mathrm{j} 59.5607$ \\
R11 & $39.9528+\mathrm{j} 29.9647$ & $39.9057+\mathrm{j} 30.0274$ & $39.9060+\mathrm{j} 30.0270$ & $-84.8999-\mathrm{j} 52.9375$ & $-84.8306-\mathrm{j} 52.9203$ & $-84.2426-\mathrm{j} 52.8063$ \\
R15 & 0 & 0 & 0 & $99.8822+\mathrm{j} 0$ & $99.8631-\mathrm{j} 0.005529$ & $99.5051+\mathrm{j} 0.2540$ \\
R16 & 0 & 0 & 0 & $-84.8999-\mathrm{j} 52.9375$ & $-84.8427-\mathrm{j} 52.9016$ & $-84.0650-\mathrm{j} 52.5711$ \\
R17 & 0 & 0 & 0 & $-84.8999-\mathrm{j} 52.9375$ & $-84.7547-\mathrm{j} 52.8574$ & $-82.0956-\mathrm{j} 51.6780$ \\
R18 & 0 & 0 & 0 & $0+\mathrm{j} 30.9635$ & $-0.005836+\mathrm{j} 30.942$ & $-0.1630+\mathrm{j} 30.6806$ \\
\hline
\end{tabular}

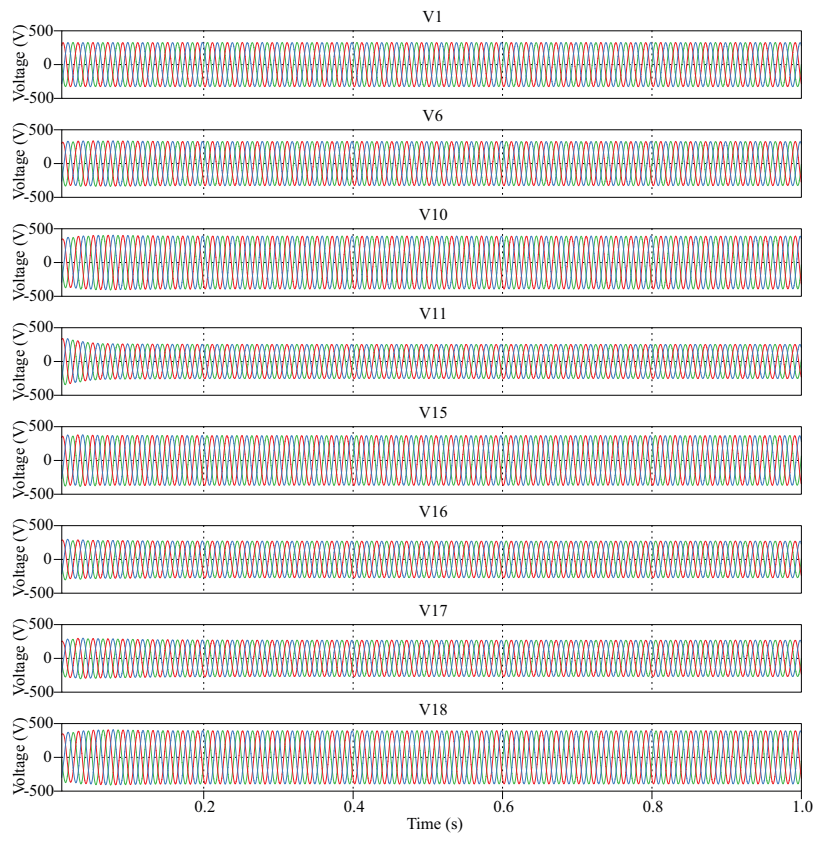

(a)

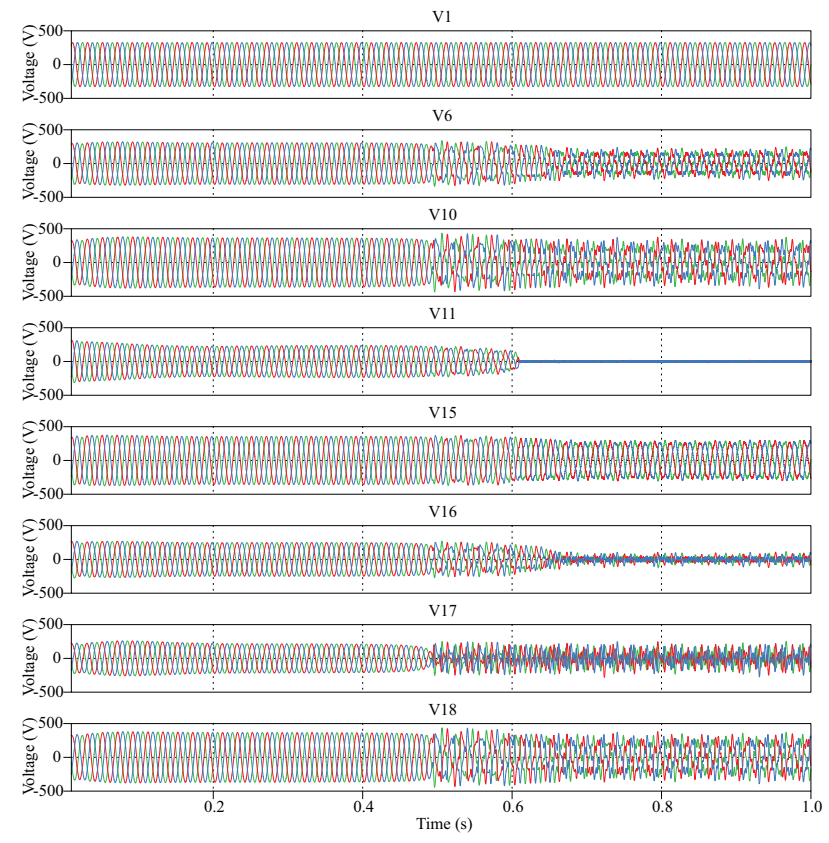

(b)

Fig. 8. Bus voltages of benchmark microgrid when different PLLs are used: (a) fast PLL case and (b) slow PLL case.

where $\omega_{n}$ and $\xi$ are the natural frequency and damping coefficient, respectively, $t_{s}$ is the settling time.

\section{B. Simulation Results}

First, the ZIP models of different buses of Fig. 7 have been evaluated, considering all the before-mentioned control and synchronization, as well as the sampling and computation delay of ADC and PWM. In this paper, $G_{P W M}(s)=e^{-0.5 s T_{s}}$ and $G_{A D C}(s)=e^{-s T_{s}}$ have been used in the models, $T_{s}$ is the sampling interval of control system. The constant impedance part of both current control and power control is

$$
Z_{k}(\text { p.u. })=8.7430 \cdot 10^{16}+j 2.2711 \cdot 10^{16} .
$$

which is much larger than all the constant impedance loads and thus can be ignored in the stability analysis.

Then, three-phase PLLs with different dynamics (bandwidth/settling time) have been utilized in the following two case studies to evaluate the influence of dynamic behaviors on the voltage stability. In the first case, PLLs of $100 \mathrm{~ms}$ settling time (bandwidth: $94 \mathrm{~Hz}$ ) have been applied to all the gridinterfaced inverters of the benchmark microgrid. In the second case, slower PLLs with $325 \mathrm{~ms}$ settling time (bandwidth: 21 $\mathrm{Hz}$ ) have been employed for the grid inverters. The constant current parts and constant power parts of two case studies have been calculated and depicted in TABLE IV. For comparison, the ideal models without synchronization have been listed in the table as well.

The case studies were simulated by using the MATLAB/Simulink environment with the aid of the PLECS toolbox. According to the scaling factor $\kappa=0.98$, the benchmark grid is supposed to be stable. Fig. 8 shows the voltage waveforms of two case studies when different PLLs are utilized. In Fig. 8(a), all the bus voltages reach the steady-state and their amplitudes stay constant. It is verified that the PLL 
with fast dynamics introduces little non-ideality on the system behavior so that the stability assessment derived from the idealized model is still valid. On the other hand, the bus voltages in Fig. 8(b) start to oscillate at around $t=0.5 \mathrm{~s}$. The voltages at Bus 11 and Bus 16 collapse at around $t=0.7 \mathrm{~s}$. Obviously, the system with a slow PLL (settling time: $325 \mathrm{~ms}$, bandwidth: $21 \mathrm{~Hz}$ ) must be considered unstable because the voltages break down. Due to the low bandwidth/slow dynamics of the PLL, there are considerable mismatches between the idealized models and the actual ZIP models of the VSCinterfaced systems, driving a theoretically stable grid to be unstable. Further investigations showed that if the system is operated under more relaxed conditions by setting the scaling factor $\kappa<0.9$, stable operation is possible even if PLLs with a very low bandwidth are used.

\section{CONCLUSION}

ZIP models of grid-interfaced VSCs taking into account both the control schemes and the PLL-based synchronization have been developed in this paper. It is shown that the numerical values obtained by taking into account the characteristics of the PLL (i.e. its bandwidth) differ significantly from what would be obtained using idealized models. As a consequence, it is not advisable to use the idealized models (ignoring the synchronization) for stability studies because this could result in a misassessment of the stability. Simulations conducted for a weak microgrid with DERs utilizing PLLs of different bandwidth demonstrate that PLLs with slow dynamics (i.e. low bandwidth) can indeed compromise the stability.

As far as three-phase ADNs are concerend, in most practical cases it is reasonable to use slow PLLs because they can attenuate grid disturbances and smoothen the output current of the VSCs. Hence, the PLL bandwidth should be limited for the sake of power quality and small-signal stability requirements, especially when the three-phase grid voltages are unbalanced and distorted [22]. In an electric network with light loading and low penetration of VSC-interfaced DERs, slow PLLs are unlikely to cause stability problems. However, as is demonstrated in this paper, using PLLs with slow bandwidth can jeopardize the stability of a weak grid. Thus, this topic deserves further attention.

\section{A CKNOWLEDGEMENT}

The research leading to these results has received funding from the European Research Council under the European Union's Seventh Framework Programme (FP/2007-2013) / ERC Grant Agreement n. [616344] - HEART, the Federal Ministry for Economic Affairs and Energy Programme [0325797A] - Entwicklung, Bau und Betrieb eines Mittelfrequenz-Mittelspannungs-MessstromErzeugers für Netzmessungen und Optimierungen, and the National Research Programme NRP70 "Energy Turnaround" of the Swiss National Science Foundation (SNSF) (For further information, please confer to www.nrp70.ch).

\section{REFERENCES}

[1] R. Majumder, "Some Aspects of Stability in Microgrids," Power Systems, IEEE Transactions on, vol. 28, no. 3, pp. 3243-3252, 2013.

[2] V. Ajjarapu and C. Christy, "The Continuation Power Flow: A Tool for Steady State Voltage Stability Analysis," Power Systems, IEEE Transactions on, vol. 7, no. 1, pp. 416-423, 1992.

[3] M. Abdel-Akher, M. E. Ahmad, R. N. Mahanty, and K. M. Nor, "An Approach to Determine a Pair of Power-Flow Solutions Related to the Voltage Stability of Unbalanced Three-Phase Networks," Power Systems, IEEE Transactions on, vol. 23, no. 3, pp. 1249-1257, 2008.

[4] I. Dobson, "Computing a Closest Bifurcation Instability in Multidimensional Parameter Space," Journal of Nonlinear Science, vol. 3, no. 1, pp. 307-327, 1993.

[5] P.-A. Löf, G. Andersson, and D. J. Hill, "Voltage Stability Indices for Stressed Power Systems," Power Systems, IEEE Transactions on, vol. 8, no. 1, pp. 326-335, February 1993.

[6] G. Irisarri, X. Wang, J. Tong, and S. Mokhtari, "Maximum Loadability of Power Systems using Interior Point Nonlinear Optimization Method," Power Systems, IEEE Transactions on, vol. 12, no. 1, pp. 162-172, 1997.

[7] J. Rocabert, A. Luna, F. Blaabjerg, and P. Rodriguez, "Control of Power Converters in AC Microgrids," Power Electronics, IEEE Transactions on, vol. 27, no. 11, pp. 4734-4749, 2012.

[8] Z. Zou, Z. Wang, and M. Cheng, "Modeling, Analysis, and Design of Multifunction Grid-Interfaced Inverters with Output LCL Filter," Power Electronics, IEEE Transactions on, vol. 29, no. 7, pp. 3830-3839, 2014.

[9] X. Wang, F. Blaabjerg, and W. Wu, "Modeling and Analysis of Harmonic Stability in an AC Power-Electronics-Based Power System," Power Electronics, IEEE Transactions on, vol. 29, no. 12, pp. 6421-6432, 2014.

[10] L. Harnefors, M. Bongiorno, and S. Lundberg, "Input-Admittance Calculation and Shaping for Controlled Voltage-Source Converters," Industrial Electronics, IEEE Transactions on, vol. 54, no. 6, pp. 3323-3334, 2007.

[11] M. Cespedes and J. Sun, "Impedance modeling and analysis of gridconnected voltage-source converters," IEEE Transactions on Power Electronics, vol. 29, no. 3, pp. 1254-1261, March 2014.

[12] C. Zhang, X. Wang, F. Blaabjerg, W. Wang, and C. Liu, "The influence of phase-locked loop on the stability of single-phase grid-connected inverter," in IEEE Energy Conversion Congress and Exposition (ECCE), Sept 2015, pp. 4737-4744.

[13] T. Van Cutsem and C. Vournas, Voltage Stability of Electric Power Systems. Springer Science \& Business Media, 1998, vol. 441.

[14] P. Kundur, Power System Stability and Control, N. J. Balu and M. G. Lauby, Eds. McGraw-Hill New York, 1994, vol. 7.

[15] G. Kron, Tensors for Circuits. Dover Publications, 1959.

[16] F. Blaabjerg, R. Teodorescu, M. Liserre, and A. V. Timbus, "Overview of Control and Grid Synchronization for Distributed Power Generation Systems," Industrial Electronics, IEEE Transactions on, vol. 53, no. 5, pp. 1398-1409, 2006.

[17] A. Timbus, M. Liserre, R. Teodorescu, P. Rodriguez, and F. Blaabjerg, "A Current-Control Strategy for Voltage-Source Inverters in Microgrids based on $H_{\infty}$ and Repetive Control," Power Electronics, IEEE Transactions on, vol. 24, no. 3, pp. 654-664, March 2009.

[18] T. Hornik and Q.-C. Zhong, "A Current-Control Strategy for VoltageSource Inverters in Microgrids based on and Repetitive Control," Industrial Electronics, IEEE Transactions on, vol. 26, no. 3, pp. 943-952, 2011.

[19] I. J. Balaguer, Q. Lei, S. Yang, U. Supatti, and F. Z. Peng, "Control for Grid-Connected and Intentional Islanding Operations of Distributed Power Generation," Industrial Electronics, IEEE Transactions on, vol. 58, no. 1, pp. 147-157, 2011.

[20] Conseil International des Grands Réseaux Électriques (CIGRÉ). Comité d'Études C6, Benchmark Systems for Network Integration of Renewable and Distributed Energy Resources. CIGRÉ, 2014.

[21] R. Teodorescu, M. Liserre et al., Grid Converters for Photovoltaic and Wind Power Systems. John Wiley \& Sons, 2011, vol. 29.

[22] P. Rodriguez, J. Pou, J. Bergas, J. I. Candela, R. P. Burgos, and D. Boroyevich, "Decoupled double synchronous reference frame pll for power converters control," IEEE Transactions on Power Electronics, vol. 22, no. 2, pp. 584-592, March 2007. 